\title{
LA FORMATION DES ENSEIGNHANTS D'ART: Lignes directrices et implicacions
}

\author{
CLAUDIA BAILAO OPA
}

Mestre em Educação, Formação e Intervenção Social, pela Université de Sorbonne - Paris

13. Graduada em Educação Artística com habilitação em Artes Plásticas (UCSal). Integra a Associação Internacional de Pesquisa Biográfica Le Sujet dans la Cité, Paris/França. ORCID: https://orcid.org/0000-0001-8899-1782. E-mail: claudiaopa@yahoo.com.br

\begin{abstract}
RESUME
Cet article présente les aspects de la recherche menée par des enseignants d'art de la municipalité de Camaçari, Bahia, dans le cadre de la maîtrise, et révèle les préoccupations qui ont émergé au cours de ce processus, à savoir: Comment la relation de plaisir et de déplaisir dans l'enseignement peut-elle affecter le travail quotidien de l'éducateur? Quelles dimensions de la subjectivité de l'éducateur sont liées à l'acte d'éduquer? Ainsi, l'objectif de ce travail est d'étudier comment la relation de plaisir et de déplaisir de l'enseignants d'art, ainsi que leurs histoires de vie en formation, peuvent résonner dans la vie quotidienne de l'école. Certains théoriciens étaient invités à cette discussion: Delory-Momberger, Barbier, Souza, Galvão, entre autres. La présente étude a un caractère qualitatif et la lumière de la recherche biographique est développée, car elle permet l'expérience de lui-même, revisitant ses origines, le sujet peut ressentir ses relations avec lui-même, avec l'autre et avec le monde. Ainsi, il cherche à connaître la réalité, sans lui
\end{abstract}

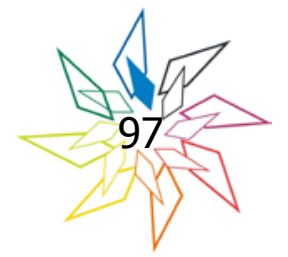


imposer aucune interposition de valeurs, motivée par la conviction selon laquelle l'espace académique se concrétise comme le lieu privilégié du débat d'idées, exempt de préjugés et de prosélytisme, vers une formation professionnelle engagée avec une discussion large et réflexive sur l'éducation contemporaine.

Mots-clés: Éducation Artistique; Formation; Recherche Biographique

\section{A FORMAÇÃO DE ARTE-EDUCADORES: DIRETRIZES E IMPLICAÇÕES}

O presente artigo relata aspectos encontrados no percurso da pesquisa de mestrado, realizada com arteeducadores do município de Camaçari, Bahia, e revela as inquietações que emergiram durante este processo, tais como: A relação de prazer e desprazer no ensinar pode repercutir no trabalho cotidiano do arte-educador? Quais dimensões da subjetividade do educador estão ligadas ao ato de educar? Sendo assim, o objetivo deste trabalho se concretiza em investigar como a relação de prazer e desprazer do arteeducador, aliada as suas histórias de vida em formação, podem fazer eco no cotidiano escolar. Alguns foram os teóricos convidados para essa discussão: Delory-Momberger, Barbier, Souza, Galvão, entre outros. O presente estudo tem natureza qualitativa e se desenvolve a luz da pesquisa biográfica, visto que possibilita a experiência de si, revisitando suas origens o sujeito pode resinificar suas relações consigo, com o outro e com o mundo. Assim, busca-se conhecer a realidade, sem nela imprimir nenhuma interposição de valores, motivado pela crença na qual o espaço acadêmico se concretiza como o lugar privilegiado para o debate de ideias, isento de preconceitos e proselitismos, rumo a uma formação profissional comprometida com uma discussão ampla e reflexiva acerca da educação contemporânea.

Palavras-clave: Arte-Educação; Formação; Pesquisa Biográfica.

\section{LA FORMACIÓN DE ARTE-EDUCADORES: DIRECTRICES E IMPLICACIONES}

El presente artículo relata aspectos encontrados en el recorrido de la investigación de maestría, realizada con arte-educadores del municipio de Camaçari, Bahia, y revela las inquietudes que surgieron durante este proceso, tales como: La relación de placer y displacer en la enseñanza puede repercutir en el trabajo de la vida cotidiana del arte-educador? ¿Cuáles dimensiones de la subjetividad del educador están ligadas al acto de educar? Siendo así, el objetivo de este trabajo se concreta en investigar cómo la relación de placer y displacer del arte-educador, aliada a sus historias de vida en formación, pueden hacer eco en el cotidiano escolar. Algunos fueron los teóricos invitados para esa discusión: Delory-Momberger, Barbier, Souza, Galvão, entre otros. El presente estudio tiene naturaleza cualitativa y se desarrolla la luz de la investigación biográfica, ya que posibilita la experiencia de sí, revisitando sus orígenes el sujeto puede resinificar sus relaciones consigo, con el otro y con el mundo. Así, se busca conocer la realidad, sin en ella imprimir ninguna interposición de valores, motivado por la creencia en la cual el espacio académico se concreta como el lugar privilegiado para el debate de ideas, exento de preconceptos y proselitismos, hacia una formación profesional comprometida con una discusión amplia y reflexiva acerca de la educación contemporánea.

Palabras clave: Arte-Educación; Formación; Investigación Biográfica. 


\section{LA FORMATION DES ENSEIGNHANTS D'ART: Lignes directrices et implicacions}

\section{Contexte}

Ce travail résulte de l'effort d'intégration des connaissances acquises lors du MERFA : Formation des adultes : champs de recherche, à l'Université Paris 13 / Sorbonne Paris Cité. À cette fin, je me propose de faire une analyse d'expérience de formation, m'appuyant pour cela sur le matériel analysé pour mon mémoire: (Im)passes Subjectives dans Éducation : histoires de vie d'enseignants d'art en formation à Camaçari (Bahia/Brésil).

Camaçari est situé à $41 \mathrm{~km}$ de Salvador, connue comme cité industrielle. Elle est la quatrième ville la plus peuplée de l'Etat de Bahia et la seconde plus grande ville de la métropole de Salvador, capitale de l'Etat. Camaçari jouit d'une grande richesse économique et naturelle. Mais malgré tout cela, la pauvreté est criante, toutes ces richesses n'ont pas été utilisées aujourd'hui pour créer de la justice sociale. Nombre de citoyens vivent à la périphérie de la ville dans un état d'extrême pauvreté.

C'est dans ce contexte que vont naître les questionnements à l'origine de ma recherche. J'y coordonne la formation des enseignants du système municipal et, de cette position, j'ai pu constater que les enseignants, malgré les bonnes conditions de travail offertes par la municipalité de Camaçari - si on les compare à celle d'autres municipalités - expriment une grande insatisfaction avec leur travail. Prise entre mon devoir de trouver des réponses pragmatiques au mal être des enseignants que je devais former et les interrogations que ce constat généraient en moi, ma recherche vise l'éloignement de mes préoccupations d'acteur vers un positionnement en intelligibilité.

\section{La Formations des enseignants d'art: lignes directrices et implications}

Pour nous, l'art est avant tout, un mot qui reconnaît aussi bien le concept de l'art que la réalité de son existence. Sans la parole, nous pourrions même douter de l'existence même de l'art. Et c'est un fait que le terme n'existe pas dans la langue de toutes les sociétés. Cependant, l'art est partout. (JANSON 1993, p. 11) 
Pour ma part, j'entends l'art comme une signature de l'existence de l'homme sur terre, où ce dernier a le rôle de raconter cette histoire là. L'homme a cette faculté de maîtriser le sujet de son travail et il va rendre compte de ce qu'il voit, entend, ressent.

Dans ce contexte, l'enseignant d'art, muni d'un cadre et de connaissances techniques va pouvoir être celui qui, va être capable de promouvoir l'expression, l'appréciation et la réflexion sur l'art - Celui qui va avoir la perception du développement de cette créativité.

La créativité n'est pas un "cadeau” donné à quelques privilégiés ; il ne se compose pas d'éclairs d'idées qui se produisent sans explication. La créativité fait partie de cette dimension symbolique $^{1}$ pouvant être développée et mise en œuvre.

Cependant, seule la connaissance artistique, même si elle est accompagnée d'expériences significatives, ne suffit pas à faire des enseignants d'art des professionnels capables d'exercer leur métier avec excellence et efficacité. Après cinq années comme coordonatrice de la formation des enseignants d'art de Camaçari, je n'ai trouvé au quotidien que des enseignants démotivés, désintéressés et se plaignant; alors je me suis mise à me poser des questions:

- Pourquoi ces enseignants qui ont accepté de participer à la formation sont autant absents ou arrivent souvent en retard?

- Pourquoi certains refusent de participer à cette formation?

Afin de nourrir ma réflexion, je suis allée chercher ce qu'il y avait derrière les lignes directrices de ces formations et le concept de professionnalisation du métier d'enseignant.

\subsection{Les Lignes directrices des formations}

Selon Jean-Marie Barbier (2011, p. 74), le champs de la formation des adultes correspond à des:

${ }^{1}$ Un terme utilisé par Jacques Lacan, à partir de 1936 pour désigner un système de représentation basé sur le langage, permettant ainsi au sujet de se référer à lui-même, consciemment et inconsciemment, dans l'exercice de son pouvoir de symbolisation (ROUDINESCO \& PLON, 1998, p. 223). 
actions de formation dont le public se caractérise par le fait qu'il se reconnaît une expérience professionnelle et/ou sociale et qu'il est déjà engagé sur cette base dans la production de représentations relatives à ses activités présentes ou futures, lesquelles finalisent directement ses engagements de formation.

C'est alors qu'on s'est mis à penser une approche spécifique adaptée aux adultes. En effet, la mentalité des adultes dans le cadre de la formation continue est bien différente de celle des étudiants du système scolaire initial. Selon Zaoré (2007, p. 5):

- Les adultes savent bien qu'on ne peut plus faire n'importe quoi, n'importe comment et à n'importe quel moment. Les adultes font appel à leurs expériences et à leur vécu pour résoudre bien des problèmes dans le cadre de leur travail;

- La personnalité de chacun, la manière de voir, de faire, change selon leurs rôle social;

- Les adultes prennent des risques calculés, ils s'engagent après avoir pesé le pour et le contre;

- Leurs motivations, besoins, sentiments, aspirations, attentes, changent et bien souvent orientent les choix individuels.

Toujours selon Zaoré (2007, p. 5-6), les différents principes de la formation pour adultes seront les suivantes:

- Le principe du respect de l'adulte en formation - tout apprenant possède ses limites d'apprentissage et sa façon d'emmagasiner des informations nouvelles. La vitesse à laquelle il assimile ces informations va jouer un rôle important dans la rétention de ces éléments;

- Le principe de la réceptivité - un apprentissage significatif commence par la volonté et le désir d'apprendre. Pour assurer cette réceptivité, l'adulte a besoin de percevoir les bénéfices de la formation et de comprendre le pourquoi de celle-ci afin de s'approprier les informations qui lui sont présentées;

- Le principe de l'interactivité - dans le cadre des adultes, l'expérience constitue la base sur laquelle s'implantent les nouveaux savoirs. Les méthodes pédagogiques employées doivent favoriser cette interactivité entre formateurs et participants à la formation;

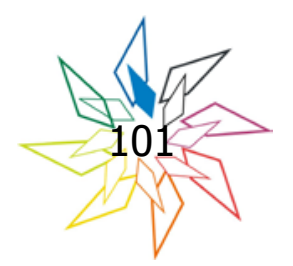


- Le principe de la facilitation de l'apprentissage - les systèmes d'apprentissage doivent respecter certains facteurs essentiels à l'acquisition des connaissances, des habiletés et des comportements. Pour ce faire, les contenus doivent être structurés et faciles à comprendre. Ils doivent faire appel à l'expérimentation et respecter un rythme adapté;

- Le principe d'acquisition et d'intégration des connaissances - il faut mesurer les acquis des apprenants à différents moments du processus de formation. En évaluant ainsi les avoirs, il est possible d'observer de manière précise les divers changements qui s'opèrent individuellement pour les sujets en formation.

En définitive, une bonne formation pour adultes, tant au niveau du contenu que de la relation formateur-apprenant, ne doit pas chercher à pallier une scolarité insuffisante, à "boucher des trous" du savoir, ou encore simplement fournir des connaissances purement théoriques. Elle doit prendre en compte l'expérience des adultes et faire appel à des méthodes d'apprentissage adaptées.

\subsection{Formation professionnelle et professionnalisante}

Selon Barbier (2011, p. 106), la professionnalisation met en scène "l'intention de transformation continue de compétences en rapport avec une intention de transformation continue d'activités". Nous pourrions dire, à sa suite, que la professionnalisation réside dans le jeu de la construction/acquisition des éléments qui vont permettre in fine d'affirmer qu'un sujet est professionnel.

Il nous semble utile ici de distinguer formation professionnelle et formation professionnalisante. Selon Wittorski (2007, p. 10), “une formation professionnelle forme son public aux outils, méthodes, techniques utiles pour agir". L'offre de formation s'emploie à proposer des situations apprenantes susceptibles de faciliter le développement d'apprentissages méthodologiques et techniques, permettant aux stagiaires de détenir progressivement les savoirs mais également les méthodes de travail leur donnant la faculté de traiter des situations professionnelles concrètes et routinières de leur activité à venir.

Par différence, une formation professionnalisante, 
(...) forme également aux outils techniques nécessaires pour agir dans l'exercice même du métier, mais cette transmission se réalise en référence aux conduites professionnelles jugées légitimes par le groupe professionnel en question. (WITTORSKI, 2007).

Une formation professionnalisante a donc pour enjeu la construction identitaire, le rapport au métier en lien avec l'identité collective. Elle nécessite la constitution préalable ou simultanée d'un groupe professionnel capable de tenir un discours sur les bonnes pratiques.

Qu'il s'agisse d'une formation professionnelle ou professionnalisante, les dispositifs proposés doivent réfléchir aux formats des situations qu'ils proposent en adéquation aux apprentissages qu'ils souhaitent développer.

La question centrale pour l'enseignant est qu'il apprenne son métier, non seulement par la formation alternée ou continue qu'il suit après son recrutement, mais aussi et surtout à travers les situations qu'il va rencontrer lors de ses premières expériences, ce qu'il est convenu d'appeler le terrain.

La problématique formulée par Wittorski (2008) considère que le développement professionnel des enseignants résulte d'une tension entre le projet de l'institution et les vécus des enseignants en formation et dans le milieu du travail. Le projet de l'institution est lisible à la fois dans les textes qui régissent la formation, dans les pratiques de formation proposées et dans les modalités d'évaluation professionnelle.

De leurs côtés, les enseignants vivent des expériences personnelles contrastées dans leur établissement d'exercice. Ils sont confrontés à des discours venant de leurs pairs, discours plus ou moins cohérents, avec le projet et les objectifs de l'institution. Dès lors, cette tension est ressentie par les enseignants en formation, tension qui selon Wittorski (2008), est à l'origine du développement des stratégies identitaires - stratégies identitaires qui seraient au cœur du processus de développement professionnel des nouveaux enseignants.

\subsection{Implications}

Eduquer, c'est amener un individu à connaître, penser, s'approprier quelque chose et se transformer. Ainsi, éduquer à travers l'art comporte un double engagement ; celui de transmettre

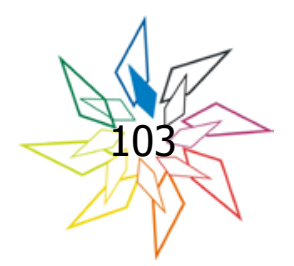


un savoir artistique, son histoire et celui de conduire l'élève à expérimenter une expression à travers ces divers langages artistiques.

Artiste dans l'âme, l'enseignant d'art est censé avoir le sens ${ }^{2}$ de la pédagogie, il aime enseigner et contribuer à l'éveil de la sensibilité et de la curiosité des plus jeunes par des travaux pratiques : dessin, peinture, bande dessinée, découverte des matériaux. Il enseigne aussi les rudiments de l'histoire de l'art et de la peinture en particulier. Accompagner les classes dans les musées et les expositions fait souvent partie de ses fonctions.

Revenons à nos questions au démarrage de cette étude.

Pourquoi les enseignants d'art ne vont pas assister avec assiduité aux formations qu'ils ont choisi? "La mission de l'enseignant d'art est de vivre l'art, de s'approprier les ressources artistiques dont il aura besoin pour transmettre cette expérience à ses élèves» (COLAGRANDE, 2010, p.12).

S'il ne s'engage pas dans le processus de formation, comment va t'il faire pour s'engager dans sa pratique?

Correia (1995, p.10) défend alors l'idée de co-investissement dans la formation. Selon lui "les efforts consentis aujourd'hui, seront récompensés demain". Quand un professeur a le pouvoir de choisir, il ne ressent pas ce sentiment d'obligation de faire la formation, il va s'engager et à l'avenir les bénéfices seront ressentis tant du côté de l'institution que de celui de l'enseignant qu'il est devenu. Pourquoi certains enseignants refusent tout bonnement de participer à ces formations? Wittorski (2005, p. 16) nous dit la chose suivante,

(...) la conception de l'efficacité du travail et les méthodes pour l'évaluer ne sont probablement pas les mêmes selon que l'on s'intéresse à un métier de l'humain,- cas de l'enseignement- ou à un métier de l'industrie par exemple. L'efficacité de l'action est une expression qui peut devenir rapidement suspecte lorsqu'on aborde l'enseignement, sans doute parce qu'elle évoque l'idée de rendement, de productivité, et au-delà, le risque de hiérarchiser les institutions, les individus et les pratiques en fonction de leur efficacité supposée.

${ }^{2}$ L'analyse du sens proposée dans cette étude proviennent des éléments de la discussion de BARBIER (2011, p. 119) "construction mentale spécifique et affects associés survenant chez un sujet à l'occasion d'une activité". 
Le risque est alors grand de ramener les objectifs et la mesure de l'efficacité de la formation à cette expression de rendement et de productivité. On oublie vite alors de valoriser les autres investissements qui demandent d'avoir une vision globale du fonctionnement de l'institution, de voir les compétences individuelles propres à chacun et les contextes socio-historiques dans lesquels les enseignants s'inscrivent. Si cette démarche n'est pas là dans le processus de la formation, les individus se démotivent et se désengagent.

\section{Recherche Biographique: fondements epistemologiques et methodologiques}

Face à la nature qualitative de cette étude qui a comme principale intention de mettre à jour émotions, pensées et comportements des enseignants, nous avons opté pour un travail basé sur la recherche biographique telle que la définit Delory-Momberger (2012, p. 06):

Le projet fondateur de la recherche biographique s'inscrit dans le cadre d'une des questions centrales de l'anthropologie sociale, qui est celle de la constitution individuelle : Comment les individus deviennent-ils des individus ? Question qui en convoque aussitôt beaucoup d'autres qui concernent le complexe de rapports entre l'individu et ses inscriptions et environnements (historiques, sociaux, culturels, linguistiques, économiques, politiques), entre l'individu et les représentations qu'il se fait de lui-même et de ses relations aux autres, entre l'individu et la dimension temporelle de son expérience et de son existencecette

De cette façon, l'objectif d'une recherche biographique est celui d'inscrire le sujet dans l'espace social propre à ses expériences, ce qui donne un sens singulier aux situations de son existence. A cette fin, la dimension temporelle se présente comme dimension constitutive de l'expérience humaine. C'est cette dimension temporelle qui par la médiation du langage va permettre de produire les écrits, les récits de vie et les narrations.

Toujours selon Delory-Momberger (2012, p. 10)

cette dimension de la temporalité de l'expérience une fois reconnue - et des approches comme celles de la psychologie sociale et de certains courants de la sociologie contemporaine la prennent tout à fait en compte -, il faut encore la spécifier sous son aspect proprement biographique au sens fort où nous l'entendons d'« écriture de la vie », d'élaboration de l'expérience,

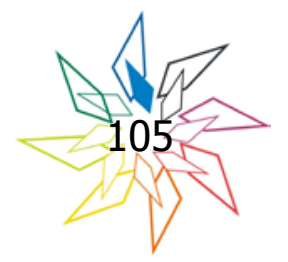


Comme le souligne Ricoeur (1983, p. 66), le récit

Est significatif dans la mesure où il dessine les traces de l'expérience temporelle » car lorsque l'on crée un tracé avec un début, un milieu et une fin. "le récit transforme alors les évènements, les actions et les gens tels qu'ils ont vécu les épisodes, (...) le récit est le lieu où l'existence humaine prend forme, où elle s'élabore et s'expérimente et va devenir une histoire. (DELORYMOMBERGER 2012, p. 12)

Le récit est ainsi le moyen mais aussi le lieu grâce auxquels l'opération de configuration ${ }^{3}$ va pouvoir se produire.

\subsection{L'acte de raconter des histoires}

Issue d'une grande tradition herméneutique selon Dilthey, Gadamer, Ricoeur et phénoménologique selon Schapp, Schütz, Berger et Luchkman, la recherche biographique comme tout recherche est orientée et n'est pas dissociable du projet et de la problématique de celui qui recherche. En même temps, la recherche bibliographique constitue un axe de réflexion épistémologique et méthodologique, tel un mouvement de pratique sociale de recherche et de formation.

L'acte de raconter son histoire de vie donne la possibilité au sujet d'organiser un dialogue intérieur quotidien à partir de moments de réflexion et de formation; il va mettre alors en évidence les expériences qu'il vit tout au long de sa vie.

Selon Morin (1980, p. 61) l'histoire de vie mobilise "l'individu de son vivant; en même temps de façon intensive, dans son lieu d'origine, et de façon extensive dans la totalité de la biosphère et c'est cette complexité que nous devons à présent affronter". Et ceci est un processus qui vise à produire lui même sa propre identité. Pineau et Le Grand $(2002$, p. 35) vont plus loin "les histoires de vie, ici définies comme la recherche et la construction des ressentis à partir de

\footnotetext{
${ }^{3}$ Terme décrit par Paul Ricoeur comme une mise en intrigue, qui va se réaliser dans et par le langage. C'est le récit, le discours qui va constituer non seulement le moyen mais aussi le lieu de cette opération : la vie est un lieu de récit et aussi la vie est là où se fait l'histoire. (DELORY-MOMBERGER, 2012, p. 40).
} 
faits temporels personnels incluent un processus d'expression et d'expérience". Cette dernière définition élargit le territoire des histoires de vie et aborde un processus humain, un phénomène anthropologique.

Dans ce contexte, Pineau et Le Grand (2012, p. 40) développent les trois raisons essentielles qui font que la vie va devenir une histoire,

- Agir, comprendre mais aussi s'émanciper. Cette trilogie de finalités des actions commence à se consolider théoriquement, de façon heuristique, lors de la formation des adultes. C'est la même distinction que fait Habermas (1976, p. 71) entre trois types de savoir: le technique, le pratique et l'émancipateur. L'émancipateur en particulier, en dehors de l'émancipation politique au sens strict, du terme, va permettre à travers l'expression de s'identifier à la libération opérée par la prise de conscience critique et réflexive des déterminants existentiels ;

- Acquérir son historicité - chercher à comprendre le sens de sa propre histoire, sa propre construction et pas seulement l'histoire des autres. Et faire jaillir la source, dans une dynamique temporelle depuis son origine ;

- Avoir accès à un présent historique singulier - l'émergence de ce cadeau singulier établit de façon synchrone un contact direct avec les facteurs déterminants de l'existence.

Toujours selon Pineau et Le Grand (2002, p. 41) "l'histoire de vie, quelque soit son utilisation est en premier lieu un acte de langage qui crée une situation socio-linguistique spécifique". Selon ces auteurs, cette situation peut se résumer de deux manières:

1. Celui qui raconte va énoncer un certain nombre de faits, d'évènements qui lui sont arrivés et il va essayer de les articuler de la manière la plus significative possible pour luimême et pour son interlocuteur. Il produit un énoncé ;

2. Même si cet énoncé est le plus clair possible, il ne sera jamais transparent : la vie ne pourra jamais être traduite en mots. Il restera toujours un résidu biologique non dit nombre de filtres personnels, sociaux, physiques existent. L'énoncé comporte pour celui qui raconte et pour l'interlocuteur un certain nombre de problèmes qui demandent une seconde opération : un travail sur l'énoncé, un travail d'analyse, d'interprétation et de critique.

Dans un premier temps, les deux acteurs travaillent en vue de trouver une distance nécessaire pour obtenir une vision compréhensive. Le narrateur, immergé dans la vie qu'il raconte, va tenter de s'en éloigner, et l'interlocuteur, loin de cette vie, doit s'en rapprocher. Arrive

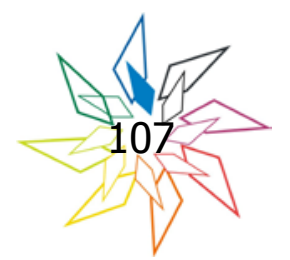


un second moment de réflexion sur l'énoncé qui va permettre de trouver un espace de savoirs associé aux expériences vécues.

\subsection{L'entretien de recherche biographique: une pratique singulière}

La conception que l'on se fait communément de la forme de l'entretien et de sa dynamique repose sur cette idée faussement simple que l'enquêteur (le chercheur) questionne et que l'enquêté (l'informateur) répond. Dans ce cadre, le questionneur habile est celui qui, ayant à la fois une connaissance suffisante du champ et un certain sens psychologique, pose les bonnes questions qui vont amener sinon les bonnes réponses, du moins les réponses bonnes, pour lui l'enquêteur. Et sans doute voit-on bien que ce modèle fonctionne lorsque l'entretien, comme c'est souvent le cas, obéit à une fonction d'illustration et de démonstration d'une (hypo)thèse posée de façon préalable et qui cherche pour se soutenir elle-même les arguments concrets que fournissent les récits de vie. (DELORY-MOMBERGER, 2012, p. 08)

L'entretien de recherche biographique est compris comme singulier, à un moment précis de l'existence. L'entretien est un des instruments de base des sciences sociales et d'une manière particulière dans le cadre de l'éducation. Les chercheurs utilisent parfois cet instrument de façon abusive. Les questions sont orientées et les réponses conformes au désir de l'enquêteur. Un des aspects fondamentaux de l'entretien de recherche biographique est l'interaction, la médiation et le climat de réciprocité entre celui qui questionne et celui qui répond. Il est nécessaire lors de l'entretien d'établir un lien d'accueil et de dialogue afin de capter ce que l'autre souhaite dire et ne souhaite pas dire. Ainsi, selon Szymanski (2004, p. 08): "durant la phase initiale de l'entretien, après la présentation de la recherche, il est bon d'avoir un petit temps de «préchaffage » pour une présentation plus personnelles afin d'établir un climat plus informel'.

C'est ce que j'ai cherché à faire plus précisément tout au long de mon travail. J'ai élaboré une feuille de route permettant d'établir ce sentiment de dialogue entre l'enquêteur et l'enquêté. J'avais les thèmes suivants à aborder : processus de scolarisation, choix de carrière et de formation initiale, formation continue, relation avec le travail au quotidien, perspectives pour le futur professionnel.

Munie de cette feuille de route, j'ai cherché les enseignants d'art et leur agenda et dans un deuxième temps, nous avons pu fixer des entretiens individuels, d'une durée moyenne d'une heure, 
que j'ai pu réaliser au sein même de l'école. Ce choix de faire les entretiens à l'école garantissait le meilleur confort pour les enseignants et offrait une ambiance qui amenait concentration et confidentialité. Les entretiens furent enregistrés et transcrits par écrit pour l'analyse ultérieure.

La sélection de ces enseignants s'est faite après avoir consulté le registre des enseignants d'art de la ville de Camaçari, en accord avec le Secrétariat à l'éducation. Les critères établis par le SEDUC ${ }^{4}$ étaient que les enseignants participants devaient avoir au moins 40 heures d'enseignement par semaine au sein de la ville et que les entretiens ne devaient pas troubler la dynamique de l'école. Suivant ces critères, cinq enseignants acceptèrent de participer aux entretiens de recherche biographique ${ }^{5}$.

Parmi les cinq professeurs d'art qui ont collaboré à cette recherche; nous avons eu quatre femmes et un homme, enseignants du collège. Les prénoms qui vont suivre ont été donné au hasard tout en gardant à l'homme un nom masculin.

Tableau 1: Tableau Synoptique de Présentation des Entretiens.

\begin{tabular}{|c|c|c|c|c|c|}
\hline & ALICE & ELISA & EDDY & MANON & MABEL \\
\hline Áge & 39 & 50 & 40 & 53 & 34 \\
\hline Expérience prof. & 14 & 16 & 7 & 17 & 8 \\
\hline $\begin{array}{c}\text { Stimulation artistique } \\
\text { durant lenfance }\end{array}$ & Oui & Non & Oui & Non & Oui \\
\hline $\begin{array}{c}\text { Ont-ils choisi leur } \\
\text { profession? }\end{array}$ & Non & Non & Non & Non & Non \\
\hline $\begin{array}{c}\text { Comment se sont-lis } \\
\text { senti pendant la } \\
\text { formation initiale? }\end{array}$ & Satisfait & Non satisfait & Satisfait & Satisfait & Satisfait \\
\hline $\begin{array}{c}\text { La première } \\
\text { expérience }\end{array}$ & Traumatisante & Tranquille & Tranquille & Tranquille & Traumatisante \\
\hline
\end{tabular}

${ }^{4}$ SEDUC - Secrétariat de l'Education de la ville de Camaçari.

${ }^{5} \mathrm{~A}$ la condition que les formulaires de consentement soient bien archivés.

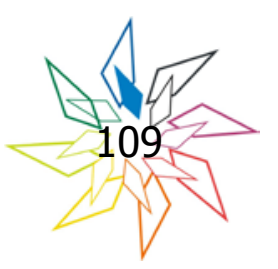




\section{L'importance des dimensions subjectives de l'experience}

La rivière qui parcourait l'arrière de notre maison était telle une vitre ramollie / Un jour, passa un homme qui déclara : ce parcours que la rivière fait ici s'appelle une baie / Il n'y eut plus alors l'image d'un serpent de verre qui parcourait l'arrière de notre maison / C'était devenu une baie / Je pense que le nom appauvrit alors l'image (BARROS 2001, p. 25),

Les plaintes des enseignants qui se manifestent dans la vie quotidienne supposent que les incertitudes sont des marques de la profession enseignante. Ces dernières nous parlent de l'existence d'autres dimensions qu'il faut prendre en compte, non seulement celle qui est objective, mais celles qui vont au-delà du sujet de la raison, où la formation des enseignants occupe une place centrale. Après tout, la formation correspond à un moment privilégié dans la construction du sens et dans le développement de la réflexivité chez les professionnels.

Pendant le temps que j'ai vécu avec les enseignants d'arts à Camaçari, j'ai connu des troubles et des affections, des charmes et des déceptions, de la persistance et de la volonté d'abandonner ce combat. J'ai réalisé que la souffrance, perçue dans leurs visages par la rugosité de l'exécution du métier d'enseignant, affiche une grâce liée à un espoir encore vivant et ferme confiance dans sa profession.

La recherche auprès d'enseignants d'art de la ville de Camaçari m'a permis de m'éloigner de mes positions d'acteur et de m'approcher d'une position d'intelligibilité de l'expérience de formation de ces enseignants. Contrairement au serpent de verre qui est devenu une baie, quitter mon pays et traverser l'océan à la recherche de nouvelles connaissances m'a enrichi énormément. Je pense que l'échange d'expériences entre professeurs et collègues de différentes formations, et la possibilité d'accès à une plus vaste bibliographie améliore ma production de façon non négligeable. Sans oublier un facteur qui ne me semblait pas pertinent auparavant mais qui aujourd'hui prend du sens : je comprends l'importance de la distance dans le travail de recherche de terrain. Selon Galvao (2012, p. 06), "La trop grande proximité avec l'autre engendre la confusion entre l'autre et soi, l'autre pouvant devenir otage de nos affects. Cet état de participation s'avère épuisant et dangereux pour notre propre survie psychique". Garder cette distance m'a permis de vivre un processus de réflexion plus ample, indispensable à mon travail, me faisant, laboratoire de moimême.

L'analyse du matériel discursif ainsi produit m'a permis de relever des catégories interprétatives permettant de mieux comprendre leur expérience de formation mais, avant tout, leur propre expérience du métier. Le tableau ci-dessous présente ces catégories. 
Tableau 1: Catégories interprétatives

\begin{tabular}{|c|l|}
\hline \multirow{2}{*}{ Ambivalence } & $\begin{array}{l}\text { Dimension affective: lien avec l'élève et } \\
\text { l'apprentissage. }\end{array}$ \\
\cline { 2 - 3 } & L'idéalisation de l'élève et l'acte d'enseigner. \\
\hline \multirow{2}{*}{ Désillusion } & Conditions de travail. \\
\cline { 2 - 3 } & Image, prestige, reconnaissance de la discipline. \\
\hline
\end{tabular}

L'ambivalence est un caractère où tendances, attitudes et sentiments contradictoires peuvent s'accumuler de façon simultanée chez l'individu. En Ornellas (2005), elle peut être aperçue dans trois domaines: celui de la Volonté - par exemple, le sujet ne veut pas manger, mais il mange; de l'Intellectuel - le sujet fait à la fois une proposition et tout son contraire; de l'Affectif - l'amour et la haine se mélangent dans la même. Pour Chemama (1995, p. 11), elle « est une disposition psychique du sujet, qui se sent ou se manifeste, sentimentalement, deux sentiments, deux activités opposées par rapport au même objet dans la même situation ».

La désillusion est caractérisée comme un état dans lequel le sujet se trouve déçu / frustré devant leurs idéalisations. Pour Florence Guist-Desprairies (2003, p. 73), elle est un

processus inhérent et nécessaire à l'évolution psychique, la désillusion est un renoncement à certains contenus idéalisés qui ne remplissent plus leur fonction étayante. Elle s'éprouve, le plus souvent, comme une déception qui se manifeste par une interprétation persécutive d'une exigence de la réalité.

Des dimensions subjectives, déclinées en "ambivalence' et "désillusion”, occupent donc une place centrale dans leur rapport au métier. Pourquoi la plupart des dispositifs de formation ne prennent pas en compte la dimension subjective de l'enseignant? Cette prise en compte serait-elle favorisée par l'introduction d'autres langages (corporel, visuel, scénique...) dans la formation ? Comment les enseignants peuvent évoluer dans ce domaine à partir de leurs expériences?

Voilà des questions auxquelles la recherche m'a amené et qui pourraient être approfondies à travers d'autres recherches pour lesquelles j'aimerais pouvoir continuer à contribuer.

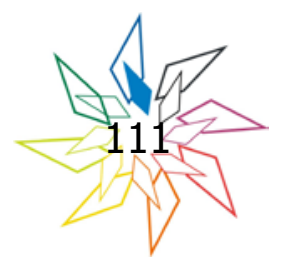




\section{REFERENNCIAS}

BARBIER J-M., GALATANU O. (dir.). Les savoirs d'action: une mise en mots des compétences? Paris: L'Harmattan. 2004.

BARBIER, J.-M. Vocabulaire d'analyse des activités. Paris : PUF. 2011

BARROS, M. O fazedor de amanhecer. Belo Horizonte: Salamandra. 2001

CHEMAMA, R. Dicionário de psicanálise. Porto Alegre : Artes Médicas. 1995

COLAGRANDE C. Arteterapia na prática. Rio de Janeiro : Wak Editora. 2010

CORREIA M. Les exclus du co-investissement. Education permanente, $\mathrm{n}^{\circ}$ 125, p. 39-56. 1995

DELORY-MOMBERGER, C. Les histoire de vie : de l'invention de soi au projet de formation. Paris : Anthropos. 2004

DELORY-MOMBERGER, C. Histoire de vie et recherche biographique en éducation, Paris : Anthropos. 2005

DELORY-MOMBERGER, C. La condition biographique. Essais sur le récit de soi dans la modernité avancée, Paris : Téraèdre. 2009

DELORY-MOMBERGER, C. Référentiels interprétatifs et modèles d'analyse. Communication présentée au 2ème Séminaire de recherche et de formation à la recherche biographique, Université Paris 13/Nord. 2012

GALVAO I. Récit d'investigation profissionnelle et émotion. Communication présentée au Colloque International « Formes d'éducation et processus d'émancipation », Université Rennes. 2012

GATTI B. A.; BARRETO E. S. S. Professores do Brasil: impasses e desafios. Brasília: UNESCO. 2009

GIUST-DESPRAIRIES, F. La figure de l'autre dans l'école républicaine. Paris : PUF. 2003

JANSON, H. W. História geral da arte. São Paulo : Martins Fontes. 1993

MORIN, E. La méthode, 2 : La vie de la vie. Paris : Le Seuil. 1980

PINEAU, G.; LES GRAND, J.-L. Les histoires de vie. Paris : PUF. 2002 
OPA C. "Arte, Educação e Psicanálise: elos de amódio que fazem eco no adolescente", dans ORNELLAS M. L. (dir). Psicanálise \& Educação. Salvador : Quarteto Editora, p. 59-72. 2011

ORNELLAS. M. L. Afetos manifestos na sala de aula. São Paulo : Annablume. 2005

RICOEUR, P. Temps ET récit I. Paris : Seuil. 1983

ROUDINESCO E.; PLON M. Dicionário de psicanálise. Rio de Janeiro: Jorge Zahar Ed. 1998

SOUZA, E. C. Autobiografias, histórias de vida e formação: pesquisa e ensino. Porto Alegre : EDPUCRS; Salvador : EDUNEB. 2012a

SOUZA, E. C. (dir.). Educação e ruralidade: memórias e narrativas (auto)biográficas. Salvador : EDUFBA. 2012b

SUÁREZ, D. H.; DÁVILA, P. V. "Documentação narrativa de experiências pedagógicas", dans SOUZA, E. C. (dir.). Educação e ruralidade: memórias e narrativas (auto)biográficas. Salvador : EDUFBA, p. 353-376. 2012

SZYMANSKI, H. A entrevista na educação: a prática reflexiva. Brasília : Liber Livro Editora. 2004

WITTORSKI R. (dir). Formation, travail et professionnalisation. Paris : 1'Harmattan. 2005

WITTORSKI R. Professionnalisation et développement professionnel. Paris : L'Harmattan. 2007

WITTORSKI R. (dir) Comment les enseignants apprennent-ils leur métier? Paris : L'Harmattan. 2008

ZAORÉ, R. Programme de formation en hygiène et salubrité. Québec : UQAR. 2007. 